Article

\title{
Investigation on Plugging and Profile Control of Polymer Microspheres as a Displacement Fluid in Enhanced Oil Recovery
}

\author{
Xiangrong Nie ${ }^{1,2, *}$, Junbin Chen ${ }^{1,2}$, Yi Cao ${ }^{1,2}$, Jinyuan Zhang ${ }^{1,2}$, Wenjing Zhao ${ }^{1,2}$, \\ Yanlong $\mathrm{He}^{1,2}$, Yunyi $\mathrm{Hou}^{3}$ and Shaomin Yuan ${ }^{4}$ \\ 1 College of Petroleum Engineering, Xi'an Shiyou University, Xi'an 710065, China; chenjbxu@126.com (J.C.); \\ caoyi3891@xsyu.edu.cn (Y.C.); 15339079834@163.com (J.Z.); wenjing5767@163.com (W.Z.); \\ stpnet@126.com (Y.H.) \\ 2 Shaanxi Key Laboratory of Well Stability and Fluid Rock Mechanics in Oil and Gas Reservoirs, \\ Xi'an Shiyou University, Xi'an 710065, China \\ 3 Research Institute of Shannxi Yanchang Petroleum (Group) Co. Ltd., Xi'an 710075, China; \\ hyyfantasy@hotmail.com \\ 4 Exploration and Development Research Institute of Daqing Oil Field; Daqing 163712, China; \\ smyuan@sina.com.cn \\ * Correspondence: nxrcup@163.com; Tel.: +86-029-897-322-68
}

Received: 20 September 2019; Accepted: 27 November 2019; Published: 2 December 2019 updates

\begin{abstract}
Polymer microspheres (PMs) are used as a new material to recover residual oil left in unswept oil areas after secondary recovery methods. The fact that the PMs plug the macropores causes the flow direction of the injection fluid to be transferred from macropores to micropores. In order to investigate the plugging and profile control mechanisms of PMs in reservoirs, four kinds of PMs with different particle sizes and four kinds of artificial cores with different permeability were selected for flooding tests, including plugging experiments and profile control experiments. The pore throat size distribution of cores was characterized by nuclear magnetic resonance (NMR) technology. The particle size distribution of PMs used in the experiment was characterized using a laser particle size analyzer. The results showed that there are six matching relationships existing simultaneously between pore throats and PMs based on theoretical analysis, which are completely plugging, single plugging, bridge plugging, smooth passing, deposition, and deformable passing. A key principle for optimizing PMs in profile control is that the particle size of the selected PMs can enter the high permeability layer well, but it is difficult for it to enter the low permeability layer. The results of this paper provide a theoretical basis for the optimal particle size of PMs during the oil field profile control process.
\end{abstract}

Keywords: polymer microspheres; profile control; enhanced oil recovery; pore throat; plugging

\section{Introduction}

Once the oil field is in the high water-cut stage, oil-water distribution in the reservoir becomes complicated, and reservoir heterogeneity becomes severe, causing water to bypass small pores and low-permeability layers, flowing along the large pores and high-permeability layers. As a result, the remaining oil in the small pores and the low-permeability strips cannot be displaced, forming an ineffective water circulation $[1,2]$. In order to further enhance oil recovery (EOR), it is possible to increase the sweep factor and oil displacement efficiency of the injected fluid. Profile control technology expands the sweep factor of the injected fluid by plugging the high permeability layer [3-5]. However, the traditional profile control agent only works in the near-well zone, and the subsequent 
injection of water quickly bypasses the plugged area and re-enters the high-permeability layer [6]. Therefore, a variety of in-depth profile control and flooding (PCF) techniques were proposed [7-9], including weak gels [10], colloidal dispersion gels [11], alkaline soil [12], foam [13], microbial [14], and oily sludge [15]. In order to further improve the in-depth PCF effect of the reservoir, scholars have proposed the idea of using polymer microspheres (PMs) for in-deep PCF.

The PMs are characterized by easy injection, good plugging, easy migration, and no damage to the reservoir. The PMs have small initial particle size and high dispersibility and are swelled in water after expansion in the reservoir [16-19]. When the PMs pass through the reservoir rock throat, the throat is blocked by a single or multiple microsphere bridges, forcing the deep liquid flow to enter the hypotonic zone and filling the reservoir with higher oil saturation. At the same time, the microspheres have good elasticity, can break through the plugging, and continue to block the reservoir to aid the redistribution of the liquid flow and the modifier [20-24]. The matching the relationship between the size of PMs and pore throats has played a particularly significant role in profile control and oil displacement $[25,26]$. When the injected PMs have a relatively large particle size, they only block the oil layer near the water injection well. The injected water bypasses the PMs and enters the high permeability layer again from the hydraulically connected low permeability layer, which leads to a poor effect of deep profile control. When the particle size of the PMs is too small, it is difficult to block the high permeability layer. Therefore, many researchers have conducted studies on optimizing the particle size of the polymer microspheres to increase the efficiency of PMs flooding [27-31].

As seen in Figure 1, previous studies have taken the pore throat size of the core and the particle size of PMs as fixed values [28,32]. In fact, the pore structure of the core is very complex, with different pore throat sizes [33-35]. Moreover, the size of PMs produced on a large scale by industrialization is not uniform $[36,37]$. Therefore, when PMs of different sizes are injected into cores with different pore sizes, multiple matching patterns will appear. Therefore, one of the purposes of this paper is to establish a more comprehensive matching mode between PMs and pore throats. In addition, the predecessors evaluated the profile control ability of PMs through a large number of physical simulation experiments and obtained some qualitative knowledge [38-41]. However, there is a lack of detailed description of the intrinsic mechanism of profile control performance of PMs. Therefore, the second purpose of this paper is to describe in detail the internal mechanism of polymer microspheres' profile control from a mesoscopic perspective. In this paper, we conducted core flooding experiments and theoretical analysis to achieve the two aims. First, the physical properties of PMs with four particle sizes were characterized. Then, the pore throat distribution of core samples used for flooding experiments was characterized by NMR. In addition, flooding experiments were conducted, including the blocking rate experiments and profile control experiments. In the end, we comprehensively analyze the above experiments to reveal the plugging and profile control mechanisms.

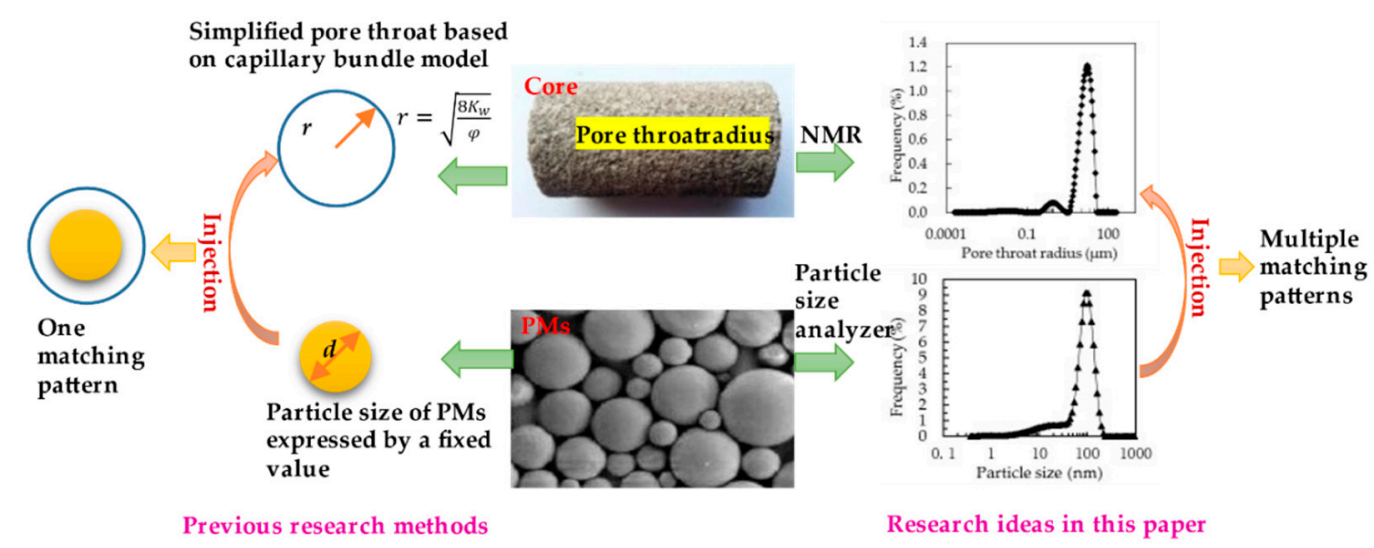

Figure 1. Sketch of previous research methods and research ideas in this paper. 


\section{Experimental Section}

\subsection{Materials}

The properties of simulated formation water (SFW) used in this paper are indicated in Table 1. Before experimentation, the SFW is filtered through a membrane filter with $0.45 \mu \mathrm{m}$ pore size.

Table 1. Properties of simulated formation water.

\begin{tabular}{ccccccccccc}
\hline \multirow{2}{*}{$\mathbf{p H}$} & \multicolumn{4}{c}{ Cation $(\mathrm{mg} / \mathrm{L})$} & \multicolumn{3}{c}{ Anion $(\mathbf{m g} / \mathbf{L})$} & \multirow{2}{*}{ Total } & Water Type \\
\cline { 2 - 9 } & $\mathbf{K}^{+}$ & $\mathbf{N a}^{+}$ & $\mathbf{C a}^{2+}$ & $\mathbf{M g}^{2+}$ & $\mathbf{B a}^{2+}$ & $\mathbf{S r}^{2+}$ & $\mathbf{H C O}^{3-}$ & $\mathbf{C l}^{-}$ & Salinity(mg/L) & \\
\hline 7.31 & 2643 & 2711 & 241 & 42 & 55 & 61 & 313 & 8641 & 14707 & $\mathrm{CaCl}_{2}$ \\
\hline
\end{tabular}

The PMs used in the experiments were prepared by inverse emulsion polymerization and supplied by Xi'an Changqing Chemical Group Co., Ltd. The kind of the PMs is non-ionic, and the relative molecular weight is about $1.76 \times 10^{7}$. The concentration of the PM solution used in the following experiments is $2000 \mathrm{mg} / \mathrm{L}$. Four PMs with different particle diameter were used, including CQ1, CQ2, CQ3, and CQ4. In order to get the PM solution used in the following experiments, $1 \mathrm{~mL}$ PM emulsion was dissolved in $500 \mathrm{~mL}$ SFW using pipette to get $2000 \mathrm{mg} / \mathrm{L} \mathrm{PM}$ solution, followed by stirred $10 \mathrm{~min}$ at $282 \mathrm{rpm}$ until the microspheres evenly dispersed in the solvent using a multihead magnetic heating agitator. The macroscopic morphology of PM emulsion with a small particle size is a light-yellow liquid, and the large particle sizes are a milky white liquid. The formed PM solutions were all white liquids (Figure 2).

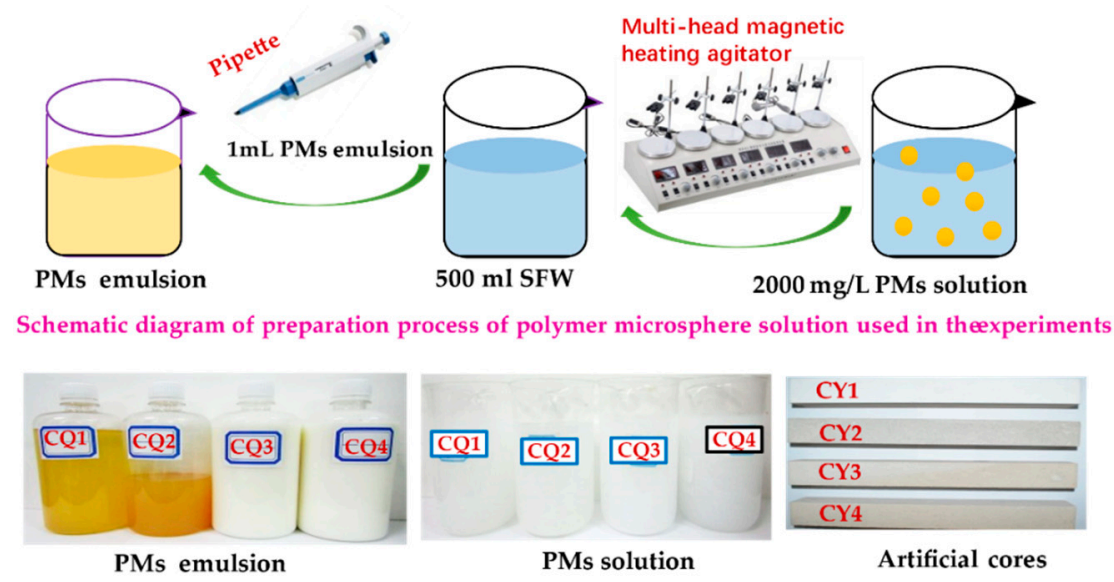

Figure 2. Schematic diagram of the preparation process of polymer microsphere solution and photographs of artificial cores and polymer microspheres used in the experiment.

The artificial cores made of epoxy resin bonded with quartz sand were used in the experiments supplied by China University of Petroleum (Beijing) (Figure 2), which were made into rectangular cores (300 mm length $\times 45 \mathrm{~mm}$ width $\times 45 \mathrm{~mm}$ height) to be used in flooding experiments. Four kinds of artificial cores with different physical properties are displayed in Table 2.

Table 2. Petrophysical properties of core samples.

\begin{tabular}{ccccc}
\hline Sample No. & CY1 & CY2 & CY3 & CY4 \\
\hline Porosity $(\%)$ & 28.87 & 23.08 & 15.81 & 5.11 \\
Permeability $\left(10^{-3} \mu \mathrm{m}^{2}\right)$ & 1387.04 & 769.86 & 354.73 & 10.29 \\
\hline
\end{tabular}




\subsection{Characterizations}

The morphology of the PM solution was obtained with the assistance of an optical microscope (LEICA-DMLP, Germany Leica Corporation, Wiesner, Germany). A laser particle size analyzer (90Plus, Brookhaven Instruments Corporation, New York, USA) was used to determine the particle size distribution of the PMs. The pore throat radius distribution of the core samples was analyzed by a nuclear magnetic resonance (MiniMR-HTHP, Shanghai Niumag Analytical Instrument Co., Ltd., Shanghai, China).

\subsection{Core Flooding Experiments}

\subsubsection{Plugging Abilities of PMs}

A core flooding apparatus was used to evaluate the plugging abilities and profile control of PMs. The schematic diagram of the experimental setup is shown in Figure 3. All the flooding experiments in this paper were conducted at $25^{\circ} \mathrm{C}$. The fluid injection rate of all the flooding experiments was constant at $0.5 \mathrm{~mL} / \mathrm{min}$. A single core hold (No.1) was used in the experiments. The procedures were as follows: (1) Vacuum and saturate the core sample with brine, and then measure the pore volume; (2) the experimental instruments were set up as shown in Figure 3; (3) high-purity $\mathrm{N}_{2}$ was injected into the system to ensure it was properly sealed; (4) the core sample was mounted in the core holder, and the absolute permeability was determined by injecting SFW; (5) $0.2 \mathrm{PV}$ (pore volume) of the PMs system was injected into the core sample; (6) then, the SFW was injected again until the pressure remained constant.

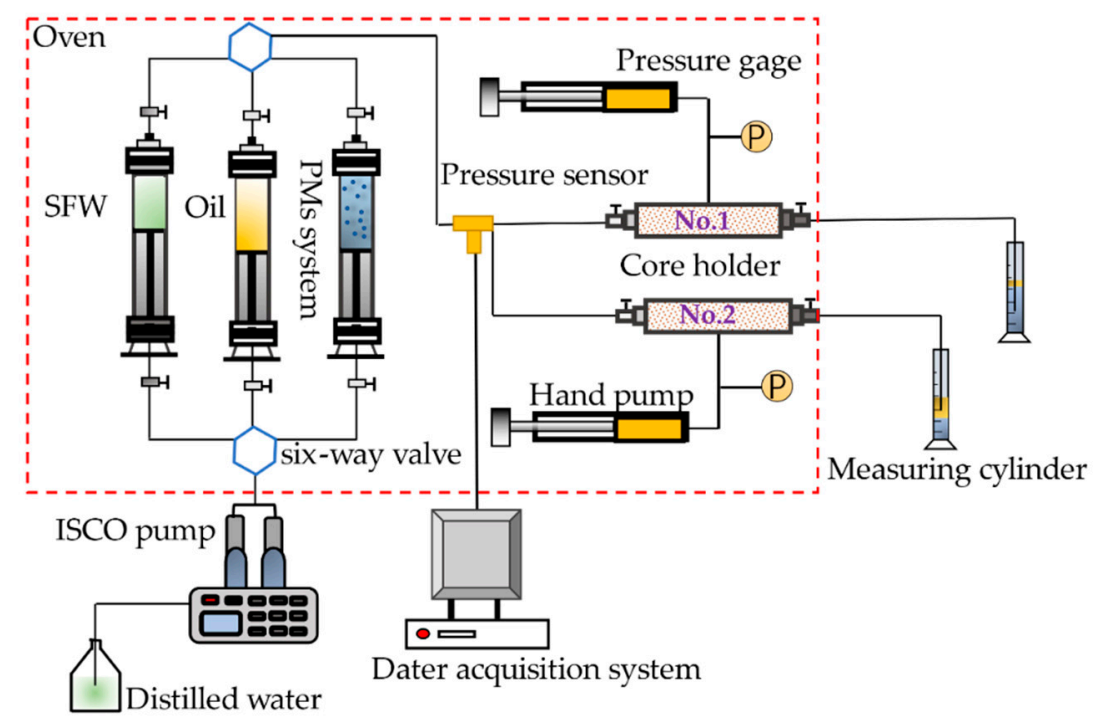

Figure 3. Schematic diagram of the core flooding apparatus.

\subsubsection{Profile Control of PMs}

Double core holds (No.1 and No.2) were used in the experiments. The specific procedures were as follows: (1) Inject 1 PV of SWF, and record the partial flow of the high and low permeability cores; (2) then, inject 1 PV of PMs system, and record the partial flow of the high and low permeability cores; (3) the SFW was injected again with an injection volume of 1.0 PV, and the partial flow of the high and low permeability cores was recorded. 


\section{Results}

\subsection{Physical Properties of the PMs}

As shown in Figure 4, the PM solution is micrograde spherical in appearance and has good sphericity. Figure 4a shows that the particle size distribution of CQ1 exhibits a single peak morphology, indicating that the particle size is relatively uniform. Figure $5 b, c$ shows that the particle size distribution of CQ2 and CO3 exhibit inconspicuous bimodal morphology, indicating that the particle size is not very uniform. Figure $5 \mathrm{~d}$ shows that the particle size distribution of CQ4 shows a distinct bimodal morphology, indicating that the uniformity of the particle size is worse than CQ2 and CQ3. The characteristic value of particle size cumulative distribution curve of PMs is shown in Table 3 , where $d_{10}, d_{50}$, and $d_{90}$ refer to the particle size when the cumulative distribution of microspheres reaches $10 \%, 50 \%$, and $90 \%$.

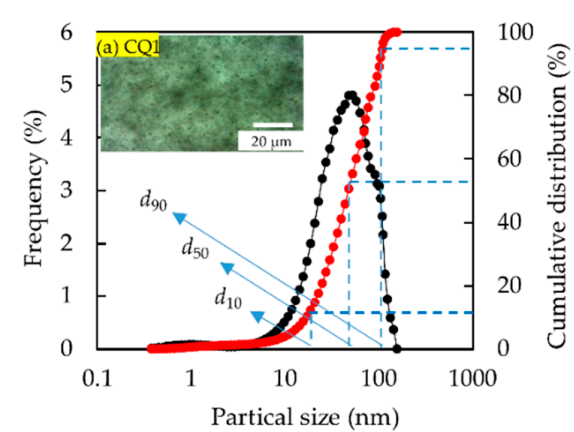

(a)

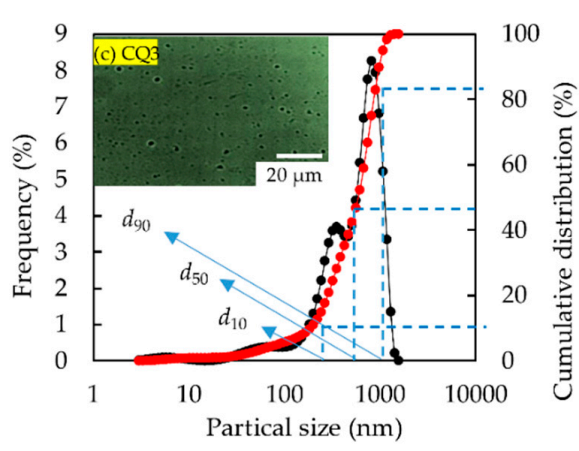

(c)

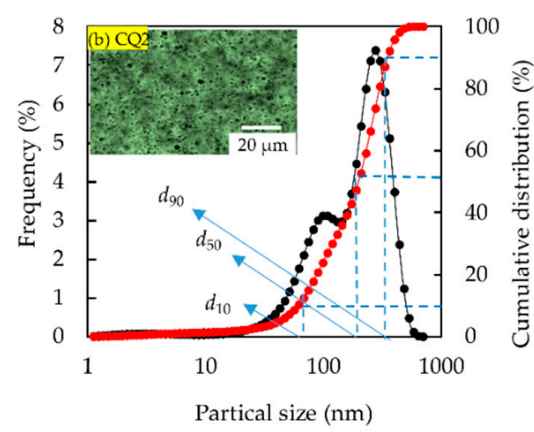

(b)

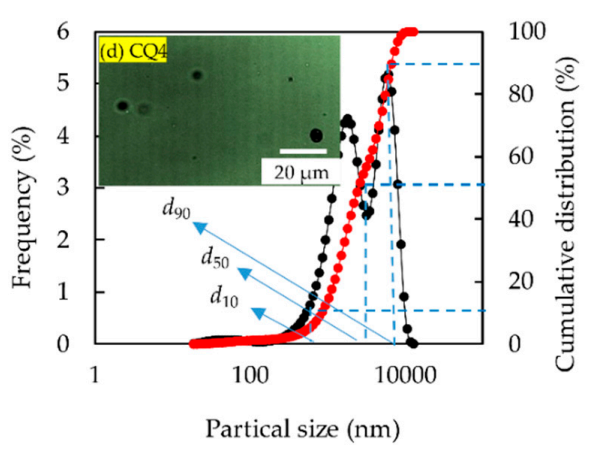

(d)

Figure 4. Morphology and particle size distribution of the PMs: (a) CQ1, (b) CQ2, (c) CQ3, and (d) CQ4.

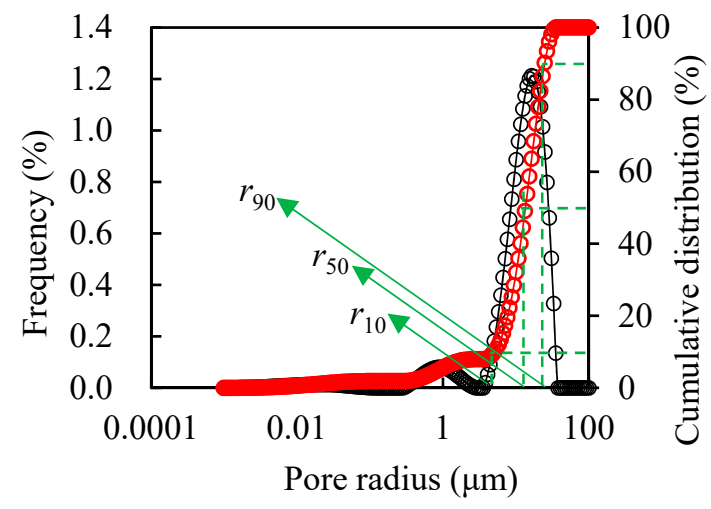

(a)

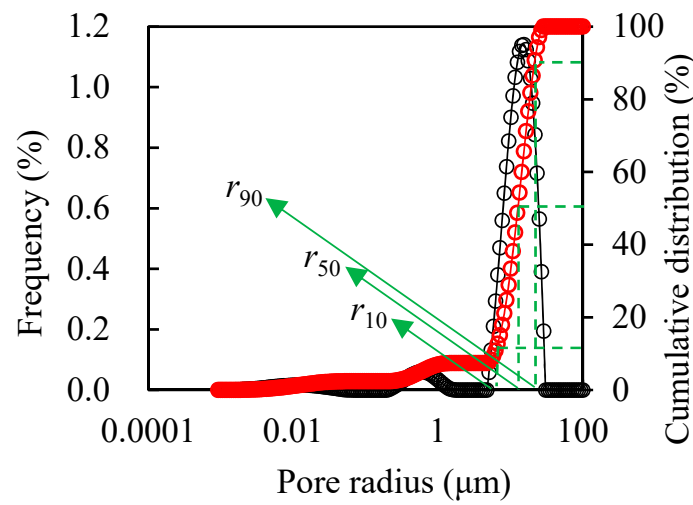

(b)

Figure 5. Cont. 


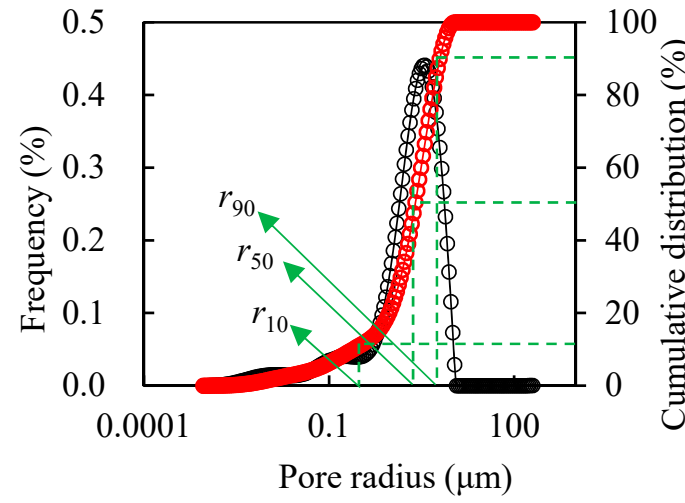

(c)

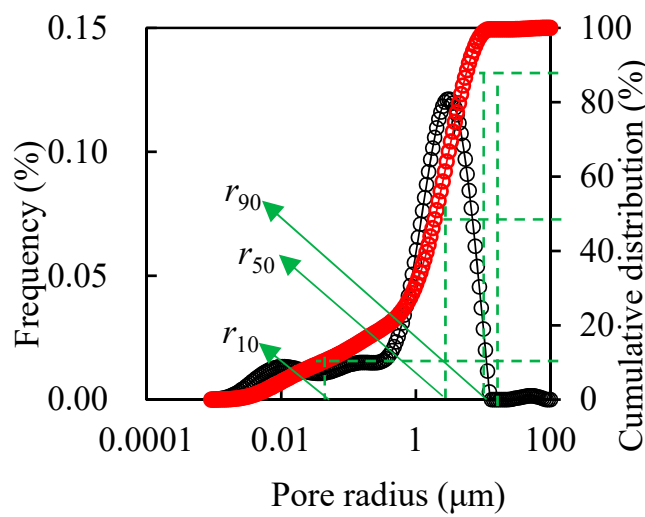

(d)

Figure 5. Pore throat distribution of cores: (a) CY1, (b) CY2, (c) CY3, and (d) CY4.

Table 3. Characteristic value of particle size cumulative distribution curve of polymer microspheres (PMs).

\begin{tabular}{cccccccc}
\hline PMs No. & $\boldsymbol{d}_{\mathbf{1 0}}(\mathbf{n m})$ & $\boldsymbol{d}_{\mathbf{5 0}}(\mathbf{n m})$ & $\boldsymbol{d}_{\mathbf{9 0}}(\mathbf{n m})$ & PMs No. & $\boldsymbol{d}_{\mathbf{1 0}}(\mathbf{n m})$ & $\boldsymbol{d}_{\mathbf{5 0}}(\mathbf{n m})$ & $\boldsymbol{d}_{\mathbf{9 0}}(\mathbf{n m})$ \\
\hline CQ1 & 17.183 & 47.939 & 104.748 & CQ3 & 181.852 & 611.402 & 974.696 \\
CQ2 & 62.121 & 208.857 & 365.511 & CQ4 & 859.160 & 2631.32 & 6687.423 \\
\hline
\end{tabular}

\subsection{Pore Throat Distribution of Cores}

Figure 5a-d illustrates the pore throat distribution of CY1, CY2, CY3, and CY4, exhibiting a single peak morphology, indicating that the pore throat of the artificial cores is relatively uniform. The characteristic value of pore radius cumulative distribution curve of cores is shown in Table 4, where $r_{10}, r_{50}$, and $r_{90}$ refer to the pore size when the cumulative distribution of cores reaches $10 \%$, $50 \%$, and $90 \%$.

Table 4. Characteristic value of pore radius cumulative distribution curve of cores.

\begin{tabular}{cccccccc}
\hline Core No. & $r_{\mathbf{1 0}}(\mu \mathrm{m})$ & $r_{\mathbf{5 0}}(\boldsymbol{\mu m})$ & $\boldsymbol{r}_{\mathbf{9 0}}(\boldsymbol{\mu m})$ & Core No. & $\boldsymbol{r}_{\mathbf{1 0}}(\boldsymbol{\mu m})$ & $\boldsymbol{r}_{\mathbf{5 0}}(\mu \mathrm{m})$ & $\boldsymbol{r}_{\mathbf{9 0}}(\mu \mathrm{m})$ \\
\hline CY1 & 5.410 & 13.339 & 24.917 & CY3 & 0.255 & 2.520 & 6.216 \\
CY2 & 6.215 & 12.445 & 21.687 & CY4 & 0.042 & 2.047 & 6.216 \\
\hline
\end{tabular}

\subsection{Plugging Abilities of PMs}

Core flooding experiments were carried out to evaluate the plugging abilities of PMs in artificial cores. The results are shown in Figure 6. The injection process can be divided into three injection stages: water flooding (WF) stage, PM flooding (PMF) stage, and finally, subsequent water flooding (SWF) stage after injection of a slug of PMs. The CQ4 PMs were injected into the CY3 and CY4 core samples, and the pressure increased sharply beyond the preset pressure, thus stopping the injection (Figure 6c,d). In the WF stage, the differential pressure kept constant. Then, in the PMF stage, the differential pressure sharply increased and then fluctuated with an approximately 1 PV PM system being injected into core samples. The reason for this test phenomenon is that when the PMs block the pore throat, the pressure rises to a maximum value, and then as the pressure difference increases, the polymer microspheres successfully pass through the pore throat, causing the pressure to drop. Then, when the next blockage occurs, the pressure difference continues to rise, and this process repeats, again and again, causing pressure fluctuations. At the SWF stage, the pressure begins to decrease gradually. The more the water is injected, the more the pressure difference decreases. The pressure difference after accumulative injection of 8PV SFW is much higher than that the initial stage of water flooding, which indicates that the PMs have good plugging performance. The results showed that the microspheres can plug the pore throat by adsorption, aggregation, and bridging. 

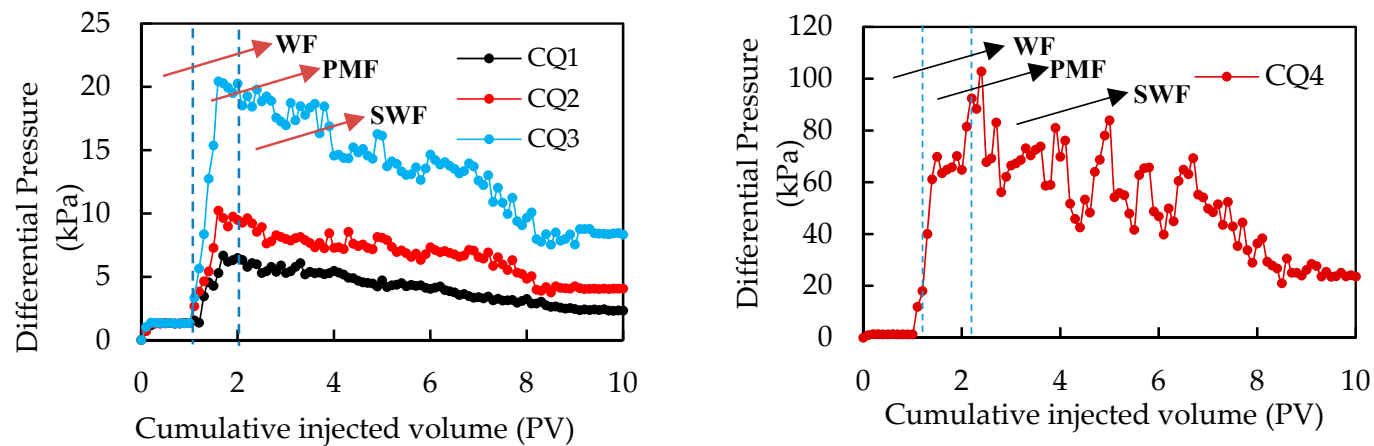

(a)
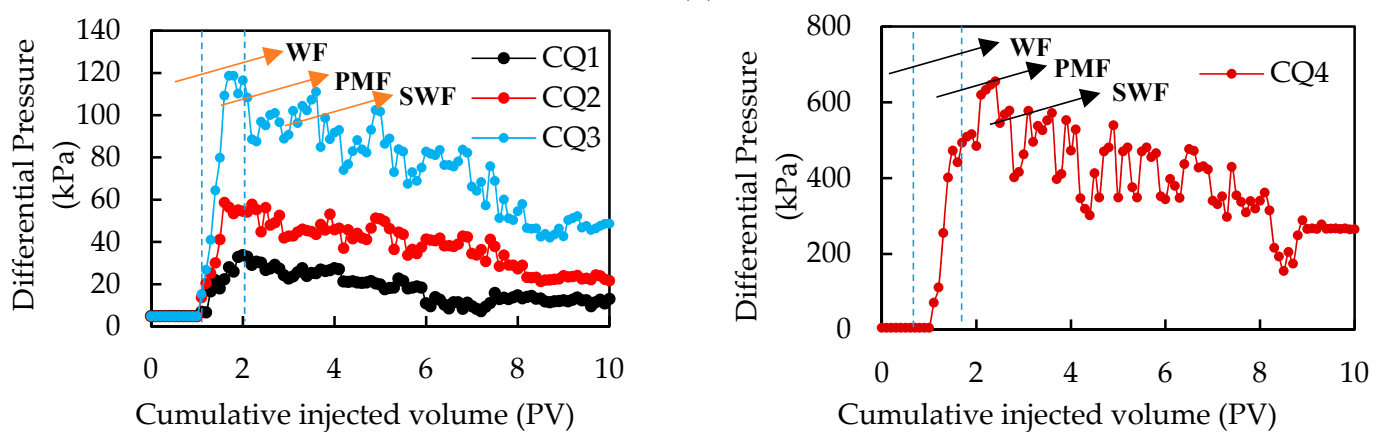

(b)
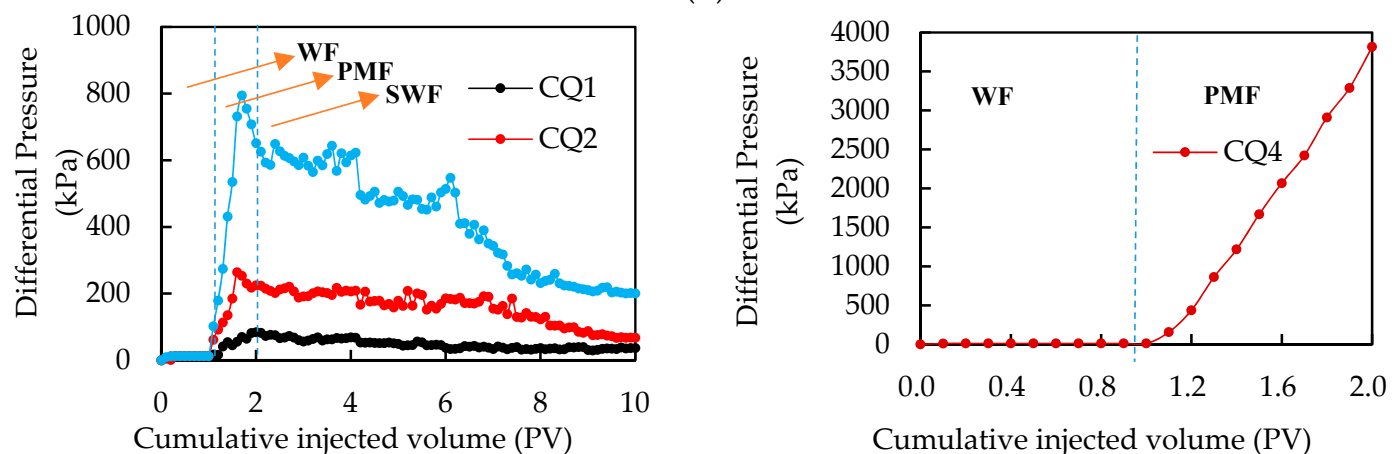

Cumulative injected volume (PV)

(c)
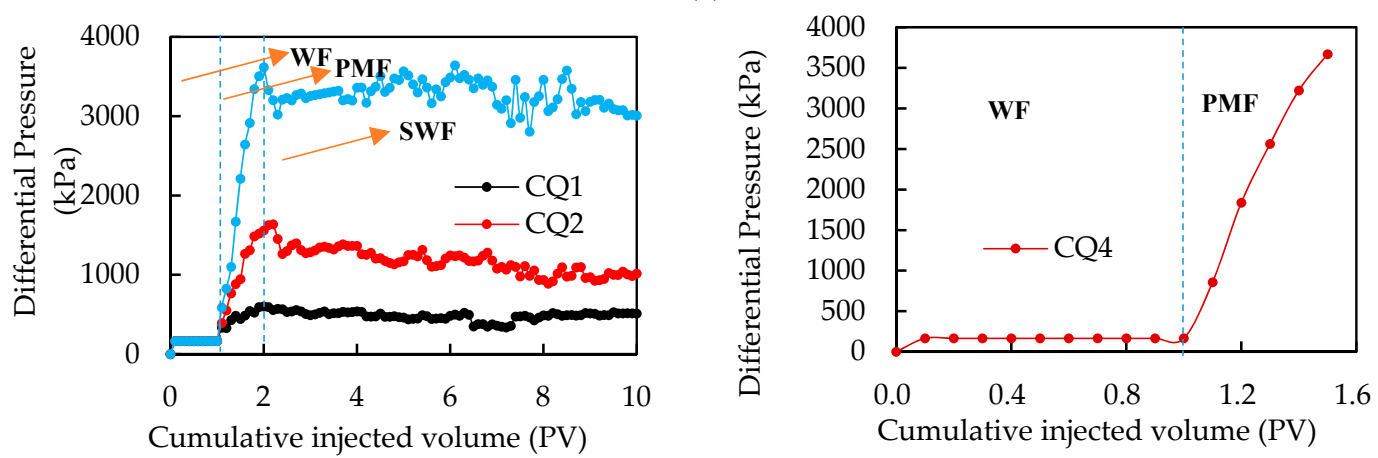

(d)

Figure 6. Pressure differential curves of PMs injected into cores: (a) CY1, (b) CY2, (c) CY3, and (d) CY4.

\subsection{Profile Control of PMs}

In profile control with PMs, there are three typical cases: (1) PMs can easily be injected into a high permeable layer, but it is difficult for them to be injected into a low permeable layer; (2) PMs can easily inject into high permeable layer and low permeable layer equally; (3) PMs can hardly be injected into a low permeable layer and high permeable layer. In order to analyze the above cases 
by experimentation, we selected PMs and cores for profile control experiments based on the results shown in Figure 6. It can be seen from Figure 6b,c that CQ4 PMs can be easily injected into the CY2 core, but it is difficult for them to be injected into the CY3 core, so we used CQ4 PMs and CY2 and CY3 cores to simulate the first typical case. Similarly, it can be seen from Figure 6b,c that CQ1 PMs can be easily injected into CY2 and CY3 cores, so we used CQ1 PMs and CY2 and CY3 cores to simulate the second case. It can be seen from Figure $6 c, d$ that it is difficult to inject CQ4 PMs into CY3 and CY4 cores, so we used CQ4 PMs and CY3 and CY4 cores to simulate the third case.

Fractional flow experiments were used to investigate the selective plugging and profile control mechanism of PMs. Figure 7a shows that in WF stage, the fractional flow of CY2 core and CY3 core is $67 \%$, and $23 \%$, respectively. During the PMF process, the fractional flow of $\mathrm{CY} 3$ core gradually increases, and the fractional flow of CY2 core gradually decreases. The fractional flow of $\mathrm{CY} 2$ core and CY3 core are both approximately $50 \%$ when the PM injection volume reaches $1 \mathrm{PV}$. The results showed that the CQ4 PMs can effectively improve the injection profile of the heterogeneous cores. However, Figure $7 \mathrm{~b}, \mathrm{c}$ shows that there is almost no change in the fractional flow curves after injecting CQ1 PMs and CQ4 PMs, respectively. In other words, CQ1 PMs and CQ4 PMs could not play a role in profile control for the $\mathrm{CY} 2$ core and $\mathrm{CY} 3$ core, and the CY3 core and CY4 core, respectively. Through a comprehensive analysis of the above three experiments, it is shown that the PMs have selective plugging properties.

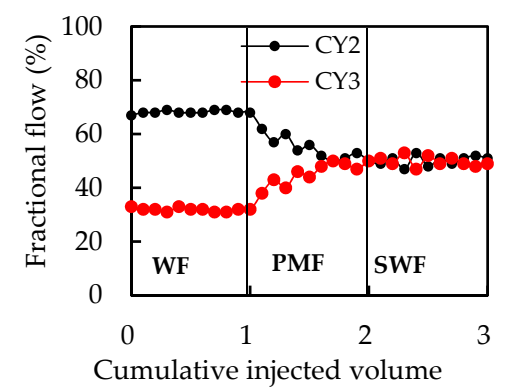

(PV)

(a)

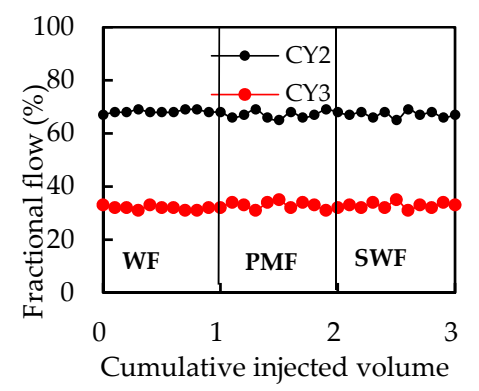

(PV)

(b)

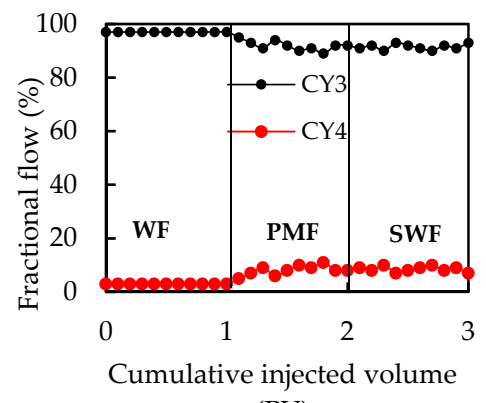

(PV)

(c)

Figure 7. Fractional flow curves in the PM injection process: (a) CQ4, (b) CQ1, and (c) CQ4.

\section{Discussion}

\subsection{Six Matching Patterns of PMs in Core}

In order to accurately characterize the matching relationship between polymer microspheres and pore throats, the pore throat radius distribution curves of four cores were transformed into pore throat diameter distribution curves, and the same diagrams were drawn with the particle size distribution curves of four polymer microspheres, as shown in Figure 8. The predecessor proposed a method for determining the matching pattern. At first, the average pore throat diameter of the core was calculated by Equation (1) [27]. Then, in combination with the size of the PMs, the matching pattern can be determined. As shown in Figure 8, the pore throat size of the core varies widely, rather than the value calculated by Equation (1). Further, the size of the PMs is not a constant value but varies within a certain range. Obviously, the method proposed by predecessors is very rough.

$$
D=2 \times \sqrt{\frac{8 K_{w}}{\varphi}}
$$

where $D$ is the average pore throat diameter of core, $\mu \mathrm{m} ; K_{w}$ is the core permeability after water flooding, $\mu \mathrm{m}^{2}$; and $\varphi$ is the porosity of the core. 


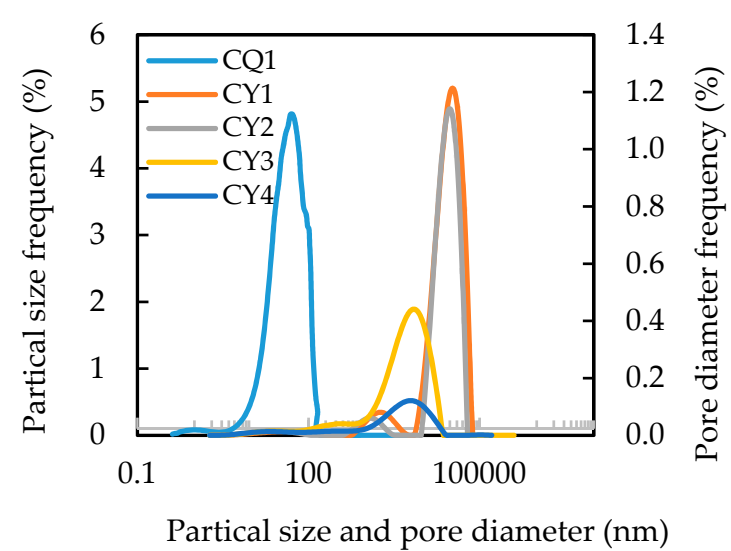

(a)

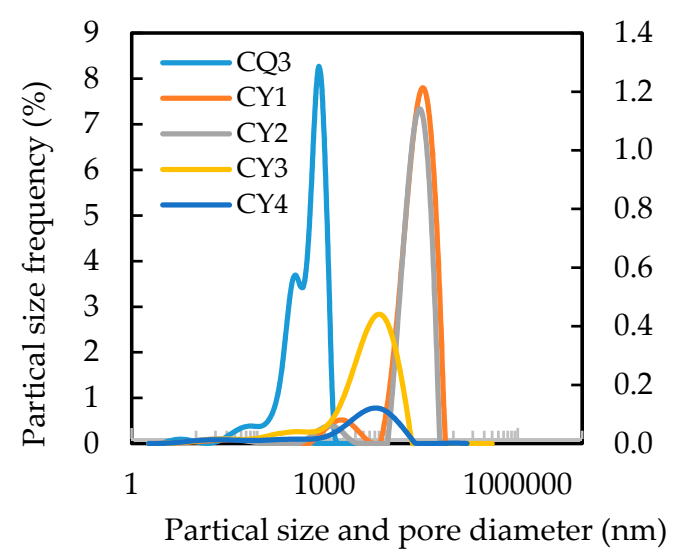

(c)

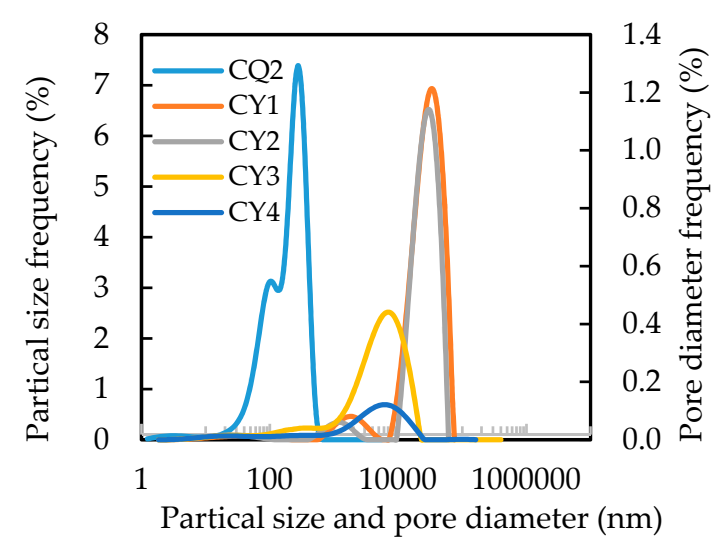

(b)

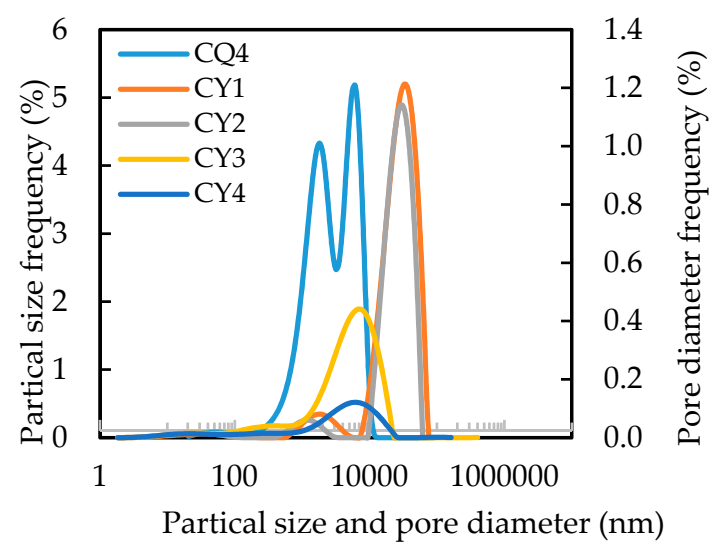

(d)

Figure 8. Diameter of pore throat and particle size of PM distribution curves: (a) CY1, CY2, CY3, CY4 and CQ1, (b) CY1, CY2, CY3, CY4 and CQ2, (c) CY1, CY2, CY3, CY4 and CQ3, (d) CY1, CY2, CY3, CY4 and CQ4.

The particle size distribution curve of CQ3 PMs and the pore throat diameter distribution curve of the CY3 core in Figure 8c were analyzed as examples. The two curves coincide partly, which indicates that the matching relationship between CQ3 PMs and pore throat is very complex. Through theoretical analysis, we summarize six typical matching models, as shown in Figure 9. Figure 9a shows that a PMs could not enter the pore since the PM size was much larger than that of the pore throat, which we name complete plugging. When the PMs and the pore throat have a close size, as shown in Figure 9b, one PM could plug the pore effectively, which we name single plugging. As pointed out in Figure 9c, some PMs formed aggregates to bridge the pore when the PMs were 2-3 times smaller than the pore, which we name bridge plugging. The PMs flow in a porous medium, they are subjected to various forces. When the resultant force is directed to the surface of the pores, the microspheres are deposited, which we name deposition, as shown in Figure 9d. The PMs could easily pass through the pores with barely any plugging, owing to their smaller size relative to the pore size, as shown in Figure 9e, which we name smooth pass. The PMs have certain deformability and can flow through the pore throat through deformation, which we name deformable passing, as shown in Figure 9f. 


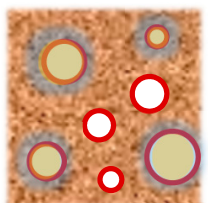

(a)

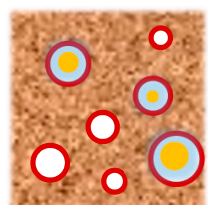

(b)

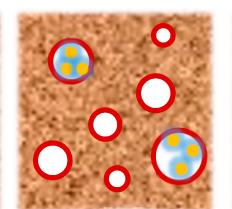

(c)

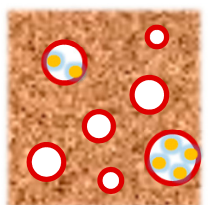

(d)

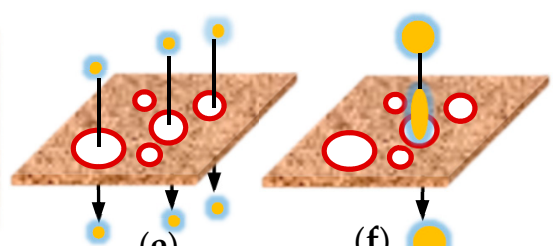

(e) (f)

Figure 9. Six plugging types of PMs in the core: (a) Complete plugging, (b) Single plugging, (c) Bridge plugging, (d) Deposition, (e) Smooth passing, (f) Deformable passing.

\subsection{Plugging Abilities of PMs in Core}

As shown in Figure 8a, the particle size distribution curve of CQ1 PMs hardly coincides with the pore throat diameter curves of the CY1 and CY2 cores, and coincides very little with the CY3 and CY4 cores. This indicates that CQ1 PMs have very weak plugging properties for CY1 and CY2 cores, and weak plugging properties for $\mathrm{CY} 3$ and $\mathrm{CY} 4$ cores. The overlap part of the curves in Figure $8 \mathrm{~b}$ is slightly larger than that in Figure 8a, indicating that CQ2 PMs have a slightly better plugging performance than CQ1 PMs. The coincidence part of the curves in Figure 8b,c increases gradually, which indicates that CQ3 PMs and CQ4 PMs have a better plugging ability, but the coincidence area of the CY4 core and CQ3 PMs and CQ4 PMs is larger, which indicates that the two PMs may be difficult to inject into the CY4 core. The same results can be obtained from Figure 6. In this work, the resistance coefficient and blocking rate were used to quantitatively characterize the plugging ability, calculated by Equations (2) and (3). The results are shown in Table 5. For the same core, the larger the particle size of PMs, the higher the resistance coefficient and blocking rate, which means the better the plugging effect. However, when the particle size of PMs exceeds the pore size of the core, it is difficult for the PMs to be injected into the core.

$$
\begin{gathered}
F_{r}=\frac{\lambda_{w}}{\lambda_{p}}=\frac{(k / \mu)_{w}}{(k / \mu)_{p}}=\frac{\Delta P_{p}}{\Delta P_{w}} \times \frac{Q_{p}}{Q_{w}} v \\
\eta=1-\frac{K_{s w}}{K_{w}}
\end{gathered}
$$

where $F_{r}$ is the resistance coefficient and $\eta$ is the blocking rate; $K_{w}, K_{p}$, and $K_{s w}$ respectively represent the core permeability after WF, PMF, and SWF, $\mu \mathrm{m}^{2} ; \mu_{w}$ and $\mu_{p}$ are, respectively, viscosity of water and $\mathrm{PM}$ system, $\mathrm{mPa} \cdot \mathrm{s} ; \Delta P_{w}$ and $\Delta P_{p}$ represent, respectively, the differential pressure of WF and PMF, MPa; and $Q_{w}$ and $Q_{p}$ are, respectively, the injection rate during $\mathrm{WF}$ and $\mathrm{PMF}, \mathrm{mL} / \mathrm{min}$.

Table 5. Evaluation of blocking abilities of PMs.

\begin{tabular}{ccccccccc}
\hline \multirow{2}{*}{ Core No. } & \multicolumn{3}{c}{ Resistance Coefficient } & \multicolumn{4}{c}{ Blocking Rate (\%) } \\
\cline { 2 - 9 } & CQ1 & CQ2 & CQ3 & CQ4 & CQ1 & CQ2 & CQ3 & CQ4 \\
\hline CY1 & 1.74 & 3.05 & 6.26 & 17.75 & 42.67 & 67.16 & 84.01 & 94.37 \\
\hline CY2 & 2.70 & 4.51 & 10.12 & 55.14 & 63.03 & 77.83 & 90.12 & 98.19 \\
\hline CY3 & 2.99 & 5.53 & 16.42 & - & 66.52 & 81.91 & 93.91 & - \\
\hline CY4 & 3.10 & 6.16 & 18.22 & - & 67.78 & 83.76 & 94.51 & - \\
\hline
\end{tabular}

\subsection{Migration Process of PMs in Core Sample}

Because the artificial core is opaque, it is difficult to observe the movement of PMs in the core directly. The typical migration process of PMs in the core can be obtained indirectly by analyzing the pressure difference curves shown in Figure 6. As shown in Figure 10, the first stage indicates that the pore throats of the core are saturated by SFW. The second stage shows that the PMs enter the core and mainly accumulate near the injection end. With the continuous injection of PMs, the third and fourth 
stage indicate that the PMs gradually migrate to the deep core, accompanied by more pore throats plugged by PMs. During subsequent water injection (stages 5, 6, and 7), some PMs flow out of the core, resulting in some blocked pore throats being dredged. With the continuous water injection, more and more PMs flow out of the core, but some of them remain in the core and play a role in profile control.

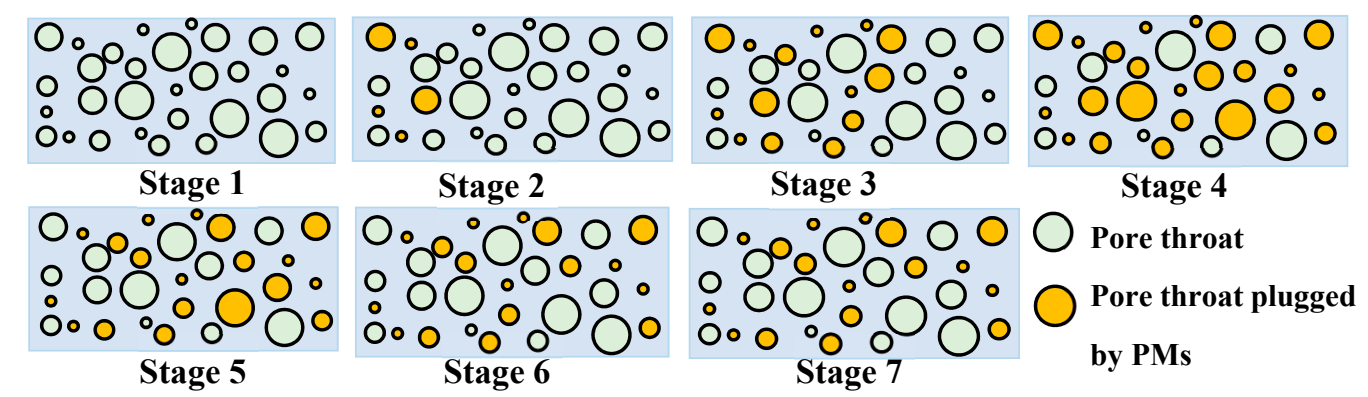

Figure 10. Typical migration diagrams of PMs in core.

\subsection{Profile Control Mechanisms}

Figure 7a shows that cq4 PMS can effectively plug the CY2 core, which results in the injection liquid transferring from the $\mathrm{CY} 2$ core to $\mathrm{CY} 3$ core, showing a good profile control effect. Figure $7 \mathrm{~b}, \mathrm{c}$ shows an invalid profile control. Combining with Figure 8, we can summarize the mechanisms of effective profile control and ineffective profile control. As shown in Figure 11a, when the particle diameter of the PMs is larger than the pore throat diameter of the high permeability layer, the PMs cannot be injected into the high permeability layer, and the profile control is invalid. As shown in Figure 11b, the PMs can enter the low permeability layer when the particle diameter of the PMs is smaller than the pore throat diameter of the low permeability layer, resulting in a decrease in the permeability of the low permeability layer, and invalid profile control. Profile control is effective only when the polymer microspheres are able to enter the high permeability layer and are unable to enter the low permeability layer, as shown in Figure 11c. That is to say, the particle diameter of the PMs is smaller than the pore throat diameter of the high permeability layer, but larger than the pore throat diameter of the low permeability layer.

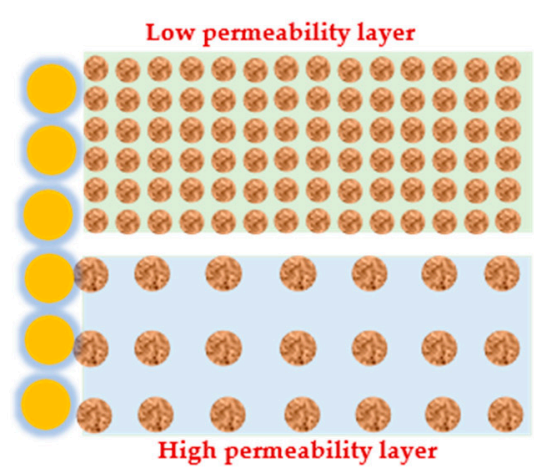

(a) Invalid profile control
Low permeability layer

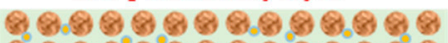

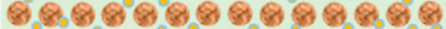

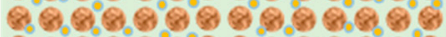
(8) ৫.

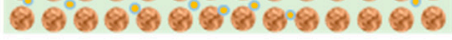

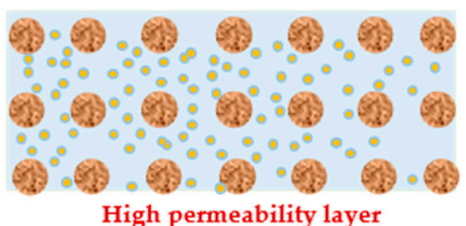

(b) Invalid profile control
Low permeability layer

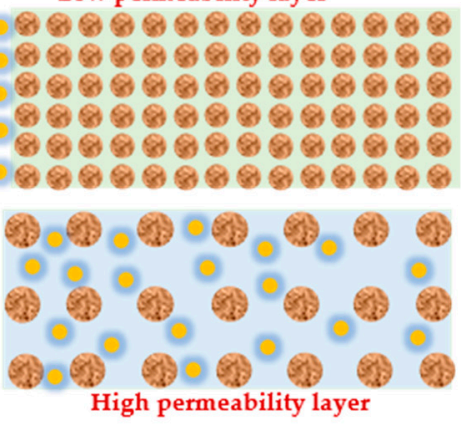

(c) Effective profile control

PMs

Sandstone particle

Figure 11. Schematic diagram of effective profile control and ineffective profile control.

\section{Conclusions}

In this work, we focused on plugging and profile control of PMs in porous media using physical experiments. The following conclusions are made:

- The particle size distribution of PMs was measured by a laser particle size analyzer, and the pore size distribution of the core was measured by NMR. The above results show that the size of PMs 
and the pore varies in a certain scale, which indicates that the previous studies were not accurate based on the average pore throat size and fixed particle size. Therefore, we point out that the particle size distribution and pore throat distribution should be fully considered in future research about plugging and migration.

- There is an overlap between the particle size distribution curve of PMs and the pore size distribution curve of the core in the same coordinate system, which shows some of the small microspheres can easily flow through the pore throat, and other of the big microspheres can hardly flow through the pore throat, resulting in various matching patterns. Based on theoretical analysis, we summarize six typical plugging types, which are completely plugging, single plugging, bridge plugging, smooth passing, deposition, and deformable passing.

- Through the plugging ability experiments, we found that plugging ability is related to the overlapping area of the $\mathrm{PMs}^{\prime}$ particle size distribution curve and the pore throat diameter distribution curve. The larger the overlapping area of the two curves, the better the plugging ability. However, it is difficult to establish the quantitative relationship between the overlapping area of the two curves and the plugging ability.

- The pressure difference decreased in the SWF stage, indicating that some PMs flowed out from the end face of the core. The typical migration stages of PMs in the core are the stage of microspheres flowing into the core, the stage of microspheres deep migration, and the stage of some microspheres flowing out of the core.

- When PMs are used for profile control, the particle size of PMs must meet certain conditions. Specifically, it is necessary to ensure that PMs can enter the high permeability layer, but not the low permeability layer.

Author Contributions: Drafting of manuscript: X.N.; acquisition of data: J.Z., W.Z., and Y.H. (Yanlong He); performance of the experiments: X.N., Y.H. (Yunyi Hou), and S.Y.; planning and supervision of the research: J.C. and Y.C.

Funding: This research was funded by National Natural Science Foundation of China (51674197; 51874239), Scientific Research Program Funded by Shaanxi Provincial Education Department (18JK0628), and Natural Science Basic Research Plan in Shaanxi Province of China (2018JQ4033, 2018JQ5208), and Shaanxi Postdoctoral Research Fund (2018BSHYDZZ53), and National Science and Technology Major Project of the Ministry of Science and Technology of China (2016ZX05047003-004). We would like to express our appreciation to the other members of the laboratory for help provided in experiments and language editing.

Conflicts of Interest: The authors declare no conflict of interest.

\section{References}

1. Zhang, C.; Qu, G.; Song, G. Formulation development of high strength gel system and evaluation on profile control performance for high salinity and low permeability fractured reservoir. Int. J. Anal. Chem. 2017, 2017, 2319457. [CrossRef] [PubMed]

2. Yu, Z.; Liu, L.; Liu, K.Y.; Yang, S.Y.; Yang, Y.Z. Petrological characterization and reactive transport simulation of a high-water-cut oil reservoir in the Southern Songliao Basin, Eastern China for $\mathrm{CO}_{2}$ sequestration. Int. J. Greenh. Gas Control 2015, 37, 191-212. [CrossRef]

3. Chen, X.; Feng, Q.; Liu, W.; Sepehrnoori, K. Modeling preformed particle gel surfactant combined flooding for enhanced oil recovery after polymer flooding. Fuel 2017, 194, 42-49. [CrossRef]

4. Ye, Z.; Xu, Y.; Chen, H.; Cheng, C.; Han, L.; Xiao, L. A novel micro-nano structure profile control agent: Graphene oxide dispersion. J. Nanomater. 2014, 2014, 582089. [CrossRef]

5. Liu, Y.; Dai, C.; Wang, K.; Zhao, M.; Gao, M.; Yang, Z.; Fang, J.; Wu, Y. Investigation on preparation and profile control mechanisms of the dispersed particle gels (DPG) formed from phenol-formaldehyde crosslinked polymer gel. Ind. Eng. Chem. Res. 2016, 55, 6284-6292. [CrossRef]

6. Sun, Q.; Zhang, N.; Li, Z.; Wang, Y. Nanoparticle-stabilized foam for mobility control in enhanced oil recovery. Energy Technol. 2016, 4, 1084-1096. [CrossRef]

7. Feng, Q.; Shi, S.; Wang, S.; Zheng, L. Numerical simulation of profile control by clay particles after polymer flooding. Pet. Sci. 2010, 7, 509-514. [CrossRef] 
8. Zhao, G.; Dai, C.; Zhao, M. Investigation of the profile control mechanisms of dispersed particle gel. PLoS ONE 2014, 9, e100471. [CrossRef]

9. Choi, S.K. pH sensitive polymers for novel conformance control and polymer flooding applications. SPE Reserv. Eval. Eng. 2008, 13, 926-939. [CrossRef]

10. Wang, W.; Gu, Y.; Liu, Y. Applications of weak gel for in-depth profile modification and oil displacement. J. Can. Pet. Technol. 2003, 42, 54-61. [CrossRef]

11. Luo, J.; Yang, H.; Wu, T.; Xiao, L. In-depth colloidal dispersion gels improve oil recovery efficiency. Glob. Geol. 2000, 3, 207-209.

12. Zhang, S.; Guo, J.; Liu, Q.; Zhang, B. The performance evaluation and application of alkali soil profile control agent. J. Oil Gas Technol. 2011, 33, 124-128.

13. Kam, S.I.; Rossen, W.R. A model for foam generation in homogeneous porous media. SPE J. 2003, 8, 417-425. [CrossRef]

14. Patel, J.; Borgohain, S.; Kumar, M.; Rangarajan, V.; Somasundaran, P.; Sen, R. Recent developments in microbial enhanced oil recovery. Renew. Sustain. Energy Rev. 2015, 52, 1539-1558. [CrossRef]

15. Liu, Y.; Li, Z.; Pan, M. A high-temperature plugging system for offshore heavy oil thermal recovery. PLoS ONE 2018, 13, e0199709. [CrossRef]

16. Yang, H.; Kang, W.; Yu, Y.; Yin, X.; Wang, P.; Zhang, X. A new approach to evaluate the particle growth and sedimentation of dispersed polymer microsphere profile control system based on multiple light scattering. Powder Technol. 2017, 315, 477-485. [CrossRef]

17. Yao, C.; Lei, G.; Li, L.; Gao, X. Preparation and characterization of polyacrylamide nano microspheres and its profile control and flooding performance. J. Appl. Polym. Sci. 2012, 127, 3910-3915. [CrossRef]

18. Wang, B.; Lin, M.; Guo, J.; Wang, D.; Xu, F.; Li, M. Plugging properties and profile control effects of crosslinked polyacrylamide microspheres. J. Appl. Polym. Sci. 2016, 133, 44-66. [CrossRef]

19. Yao, C.; Lei, G.; Gao, X.; Li, L. Controllable preparation, rheology, and plugging property of micron-grade polyacrylamide microspheres as a novel profile control and flooding agent. J. Appl. Polym. Sci. 2013, 130, 1124-1130. [CrossRef]

20. Zhu, D.; Hou, J.; Chen, Y.; Zhao, S.; Bai, B. In-situ surface decorated polymer microsphere technology for enhanced oil recovery in high-temperature petroleum reservoirs. Energy Fuels 2018, 33, 3312-3321. [CrossRef]

21. Li, J.; Niu, L.; Lu, X. Migration characteristics and deep profile control mechanism of polymer microspheres in porous media. Energy Sci. Eng. 2019, 7, 2026-2045. [CrossRef]

22. Abdulbaki, M.; Huh, C.; Sepehrnoori, K.; Delshad, M.; Varavei, A. A critical review on use of polymer microgels for conformance control purposes. J. Pet. Sci. Eng. 2014, 122, 741-753. [CrossRef]

23. Li, L.; Han, X.; Meng, L. Experimental research on profile control for oil displacement by functional polymer in low permeability fractured reservoir. Physics Procedia 2012, 25, 1292-1300. [CrossRef]

24. Bai, B.; Zhou, J.; Yin, M. A comprehensive review of polyacrylamide polymer gels for conformance control. Pet. Explor. Dev. 2015, 42, 525-532. [CrossRef]

25. Yang, H.; Kang, W.; Yin, X.; Tang, X.; Song, S.; Lashari, Z.; Bai, B.; Sarsenbekuly, B. Research on matching mechanism between polymer microspheres with different storage modulus and pore throats in the reservoir. Powder Technol. 2017, 313, 191-200. [CrossRef]

26. Lin, M.; Guo, J.; Xu, F.; Zhang, G.; Li, M.; Peng, B. Study on the matching between cross-linked polymer microspheres and nuclear-pore membranes. Adv. Mater. Res. 2010, 160, 1346-1353. [CrossRef]

27. Zhao, S.; Pu, W.; Wei, B.; Xu, X. A comprehensive investigation of polymer microspheres (PMs) migration in porous media: EOR implication. Fuel 2019, 235, 249-258. [CrossRef]

28. Li, J.; Liu, Y.; Na, Z.; Zeng, Z.; Jiang, H. Investigation on the adaptability of the polymer microspheres for fluid flow diversion in porous media. J. Dispers. Sci. Technol. 2014, 35, 120-129. [CrossRef]

29. Yu, X.; Pu, W.; Chen, D.; Zhang, J.; Zhou, F.; Zhang, R.; Gu, S. Degradable cross-linked polymeric microsphere for enhanced oil recovery applications. RSC Adv. 2015, 5, 62752-62762. [CrossRef]

30. Hendraningrat, L.; Zhang, J. Polymeric nanospheres as a displacement fluid in enhanced oil recovery. Appl. Nanosci. 2015, 5, 1009-1016. [CrossRef]

31. Pu, W.; Zhao, S.; Wang, S.; Wei, B.; Yuan, C.; Li, Y. Investigation into the migration of polymer microspheres (PMs) in porous media: Implications for profile control and oil displacement. Colloids Surf. A Physicochem. Eng. Asp. 2018, 504, 265-275. [CrossRef] 
32. Wang, J.; Liu, H.; Wang, Z.; Xu, J.; Yuan, D. Numerical simulation of preformed particle gel flooding for enhancing oil recovery. J. Pet. Sci. Eng. 2013, 112, 248-257. [CrossRef]

33. Liu, M.; Xie, R.; Guo, J.; Jin, G. Characterization of pore structures of tight sandstone reservoirs by multifractal analysis of the NMR T 2 distribution. Energy Fuels 2018, 32, 12218-12230. [CrossRef]

34. Zhou, Y.; Wu, S.; Li, Z.; Zhu, R.; Xie, S.; Jing, C.; Lei, L. Multifractal study of three-dimensional pore structure of sand-conglomerate reservoir based on CT Images. Energy Fuels 2018, 32, 4797-4807. [CrossRef]

35. Cao, Z.; Liu, G.; Zhan, H.; Li, C.; You, Y.; Yang, C.; Jiang, H. Pore structure characterization of Chang-7 tight sandstone using MICP combined with $\mathrm{N}_{2}$ GA techniques and its geological control factors. Sci. Rep. 2016, 6, 36919. [CrossRef]

36. Ming, W.; Zhao, J.; Lu, X.; Wang, C.; Fu, S. Novel characteristics of polystyrene microspheres prepared by microemulsion polymerization. Macromolecules 1996, 29, 7678-7682. [CrossRef]

37. Deng, J.; Deng, J. Optically active microspheres from helical substituted polyacetylene with pendent ferrocenyl amino-acid derivative. Preparation and recycling use for direct asymmetric aldol reaction in water. Polymer 2017, 125, 200-207. [CrossRef]

38. Feng, Q.; Chen, X.; Zhang, G. Experimental and numerical study of gel particles movement and deposition in porous media after polymer flooding. Transp. Porous Media 2013, 97, 67-85. [CrossRef]

39. Hua, Z.; Lin, M.; Dong, Z.; Li, M.; Zhang, G.; Yang, J. Study of deep profile control and oil displacement technologies with nanoscale polymer microspheres. J. Colloid Interface Sci. 2014, 424, 67-74. [CrossRef]

40. Zhou, M.; Nie, X.; Zhou, L.; Hou, L.; Zhao, J.; Yang, Y. Study of crosslinked copolymer nanospheres with temperature resistance, salinity resistance, and deep profile control. J. Appl. Polym. Sci. 2017, 134, 45131. [CrossRef]

41. Zhou, M.; Zou, J.; Gu, Y.; Yi, R.; Tu, H. Preparation of magnetic polymer nanosphere and its profile control. J. Dispers. Sci. Technol. 2019, 4. [CrossRef]

(C) 2019 by the authors. Licensee MDPI, Basel, Switzerland. This article is an open access article distributed under the terms and conditions of the Creative Commons Attribution (CC BY) license (http://creativecommons.org/licenses/by/4.0/). 